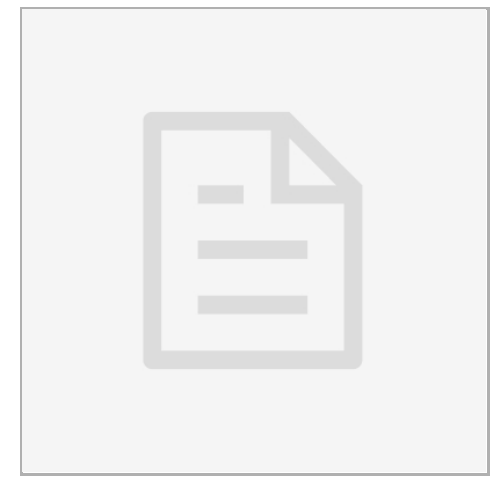

MAY 13, 2020

\title{
(3) Find proteins of unknown function (PUF) using Plantannot - Protocol C
}

Marcos

Viana $^{1}$,

${ }^{1}$ Embrapa

Mauricio Mudadu

\section{open ठaccess}

\section{DOI:}

dx.doi.org/10.17504/protocol s.io.bf88jrzw

\section{Protocol Citation: Marcos}

Viana, Adhemar Zerlotini,

Mauricio Mudadu 2020. Find proteins of unknown function (PUF) using Plantannot Protocol C . protocols.io https://dx.doi.org/10.17504/p rotocols.io.bf88jrzw

License: This is an open access protocol distributed under the terms of the Creative Commons Attribution License, which permits unrestricted use, distribution, and reproduction in any medium, provided the original author and source are credited

Protocol status: In development

We are still developing and optimizing this protocol

Created: May 11, 2020

Last Modified: May 13, 2020

PROTOCOL integer ID: 36864

\section{Entering application}


1 Enter the Plantannot Result's page, with empty filters and text box search:

https://www.machado.cnptia.embrapa.br/plantannot/find/?q=

Or you can enter the https://www.machado.cnptia.embrapa.br/plantannot initial page and click on the magnifying glass with the text box empty as well.
Plant Co-expression Annotation Resource
Data summary About
Embrapa

https://www.machado.cnptia.embrapa.br/plantannot

\section{Filtering}

2 Visualize the "Filters" card on the left of the page from step1:

\section{Filters}

Organism (53)

apply

Amaranthus hypochondriacus $(69,156)$

Amborella trichopoda $(80,538)$

Ananas comosus $(81,072)$

Aquilegia coerulea $(117,123)$

Feature type

apply

gene $(1,862,010)$

mRNA $(2,332,974)$

polypeptide $(2,332,974)$

\section{Orthology}

apply

no orthology $(4,636,180)$

orthology $(1,891,778)$

Coexpression

apply 


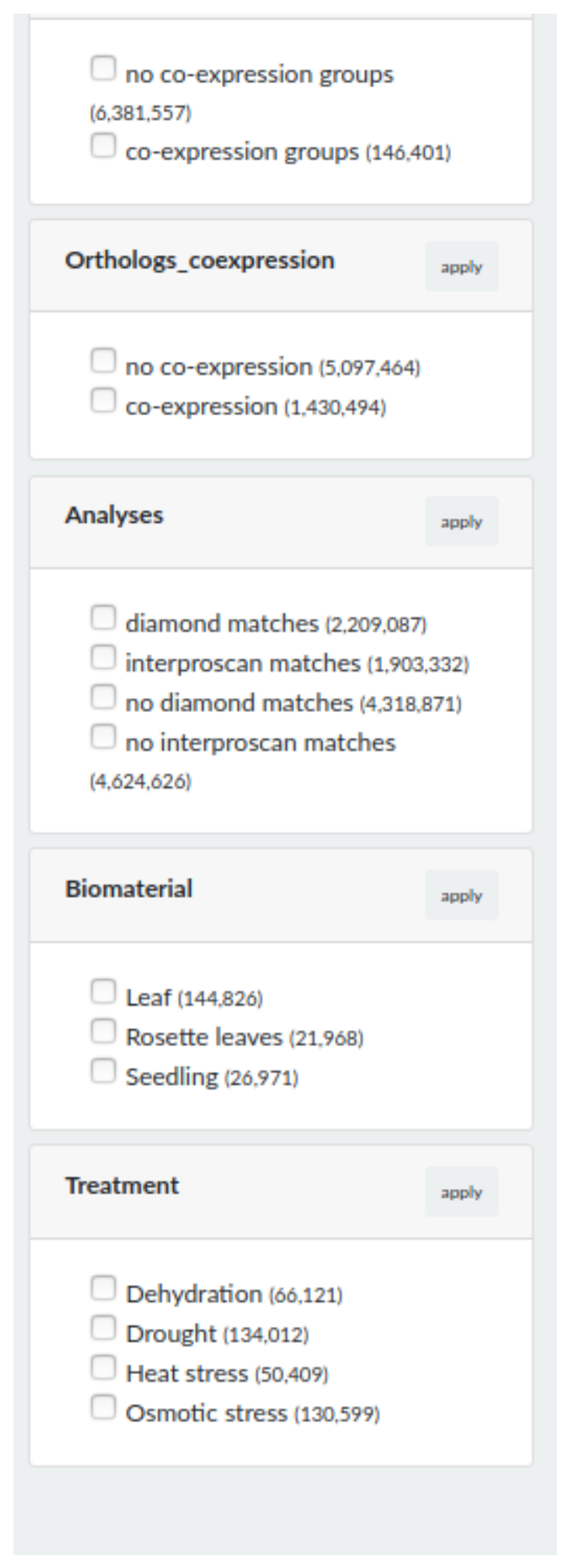

https://www.machado.cnptia.embrapa.br/plantannot/find/?q=

2.1 In the "Organisms" filter, select any organisms (expand the organism's list using the green arrow) or select all by leaving all boxes empty. We will use Oropetium tomaeum as example. Click "apply" to execute the filter: 
Amaranthus hypochondriacus

$(69,156)$

Amborella trichopoda $(80,538)$

Ananas comosus $(81,072)$

Aquilegia coerulea $(117,123)$

Arabidopsis halleri $(78,830)$

Arabidopsis lyrata $(97,337)$

Arabidopsis thaliana $(98,188)$

Boea hygrometrica $(143,334)$

Boechera stricta $(87,040)$

Brachypodium distachyon

(140.254)

Brachypodium stacei $(102,612)$

Brassica oleracea $(106,200)$

Brassica rapa $(127,232)$

Capsella grandiflora $(77,927)$

Capsella rubella $(83,415)$

Carica papaya $(83,355)$

Citrus clementina $(92,391)$

Citrus sinensis $(117,673)$

Cucumis sativus $(82,231)$

Daucus carota $(96,349)$

Eucalyptus grandis $(128,909)$

Eutrema salsugineum $(84,919)$

Fragaria vesca $(98,493)$

Glycine max $(233,338)$

Gossypium raimondii $(192,039)$

Kalanchoe fedtschenkoi $(121,344)$

Kalanchoe laxiflora $(188,815)$

Linum usitatissimum $(130,439)$

Malus domestica $(190,548)$

Manihot esculenta $(115,795)$

Medicago truncatula (175,532)

Mimulus guttatus $(95,286)$

Musa acuminata $(109,584)$

Oropetium thomaeum (85.338)

Oryza sativa $(147,037)$

Panicum hallii $(136,936)$

Panicum virgatum $(348,885)$

Phaseolus vulgaris $(101,423)$

Populus trichocarpa $(187,361)$

Prunus persica $(121,051)$

Ricinus communis $(93,663)$

Salix purpurea $(160,905)$

Setaria italica $(120,586)$

Setaria viridis (132.402)

Solanum lycopersicum $(104,175)$ 
Zostera marina $(61,350)$

https://www.machado.cnptia.embrapa.br/plantannot/find/? $q=\&$ selected_facets=organism\%3AOropetium+thomaeum

2.2 In the "Feature type" filter, select "polypeptide", and click "apply" to execute the filter:

Feature type

gene $(1,862,010)$

mRNA $(2,332,974)$

$\checkmark$ polypeptide $(2,332,974)$

https://www.machado.cnptia.embrapa.br/plantannot/find/? $q=\&$ selected_facets=organism\%3AOropetium+thomaeum\&selected_facets=so_term\%3Apol ypeptide

2.3 In the Orthology filter, select "orthology", and click "apply" to execute the filter: 
$\checkmark$ orthology $(18,755)$

https://www.machado.cnptia.embrapa.br/plantannot/find/? $q=\&$ selected_facets=organism\%3AOropetium+thomaeum\&selected_facets=so_term\%3Apol ypeptide\&selected_facets=orthology\%3A1

2.4 In the "Orthologs_coexpression" filter, select "co-expression", and click "apply" to execute the filter:

Orthologs_coexpression

apply remove

no co-expression (0)

$\checkmark$ co-expression (15,641)

https://www.machado.cnptia.embrapa.br/plantannot/find/?

$q=\&$ selected_facets=organism\%3AOropetium+thomaeum\&selected_facets=so_term\%3Apol ypeptide\&selected_facets=orthology\%3A1\&selected_facets=orthologs_coexpression\%3Atru $\underline{\mathrm{e}}$

2.5 In the "Analyses" filter, select both "no diamond matches" and "no interproscan matches", and 
Analyses

apply remove

diamond matches $(0)$

$\square$ interproscan matches (0)

$\nabla$ no diamond matches (10)

$\checkmark$ no interproscan matches (10)

https://www.machado.cnptia.embrapa.br/plantannot/find/?

$q=\&$ selected_facets=organism\%3AOropetium+thomaeum\&selected_facets=so_term\%3Apol ypeptide\&selected_facets=orthology $\% 3 A 1 \&$ selected_facets=orthologs_coexpression\%3Atru e\&selected_facets=analyses\%3Ano+diamond+matches\&selected_facets=analyses\%3Ano+i nterproscan+matches

2.6 Leave the "Coexpression" filter empty:

Coexpression

apply

https://www.machado.cnptia.embrapa.br/plantannot/find/? $\mathrm{q}=\&$ selected_facets=organism\%3AOropetium+thomaeum\&selected_facets=so_term\%3Apol ypeptide\&selected_facets=orthology $\% 3 A 1$

2.7 Leave the "Biomaterial" and "Treatment" filters empty: 


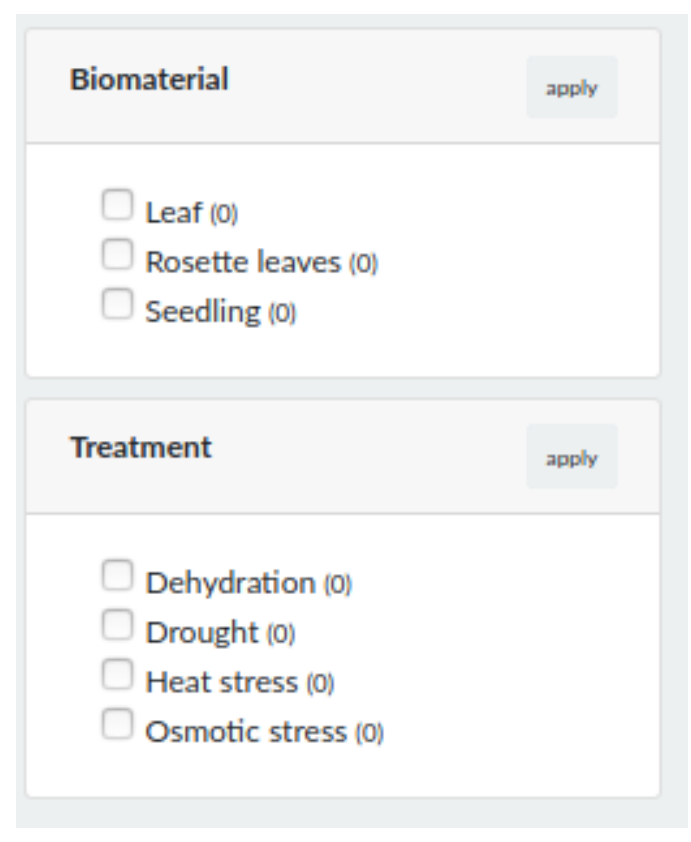

https://www.machado.cnptia.embrapa.br/plantannot/find/? q=\&selected_facets=organism\%3AOropetium+thomaeum\&selected_facets=so_term\%3Apol ypeptide\&selected_facets=orthology\%3A1\&selected_facets=orthologs_coexpression\%3Atru e\&selected_facets=analyses $\% 3 A$ no+diamond+matches\&selected_facets=analyses $\% 3 A n o+i$ nterproscan+matches

3 After execution of all filters we will have the following list of filters:

\section{Selected filters}

organism:Oropetium thomaeum $\mathbf{X}$

so_term:polypeptide $\mathbf{X}$

orthology $\mathbf{x}$

coexpression in orthologs $\mathbf{X}$

analyses:no diamond matches $\mathbf{X}$

analyses:no interproscan matches $\mathbf{X}$

https://www.machado.cnptia.embrapa.br/plantannot/find/? $\mathrm{q}=\&$ selected_facets=organism\%3AOropetium+thomaeum\&selected_facets=so_term\%3Apolype ptide\&selected_facets=orthology\%3A1\&selected_facets=orthologs_coexpression\%3Atrue\&sele cted_facets=analyses\%3Ano+diamond+matches\&selected_facets=analyses\%3Ano+interprosc an+matches 


\section{Viewing results}

$4 \quad$ Visualize the "Results" card on the center-right of the screen, we will have the resulting list of Oropetium's PUFs supposedly related to abiotic stress by using orthology and co-expression networks:

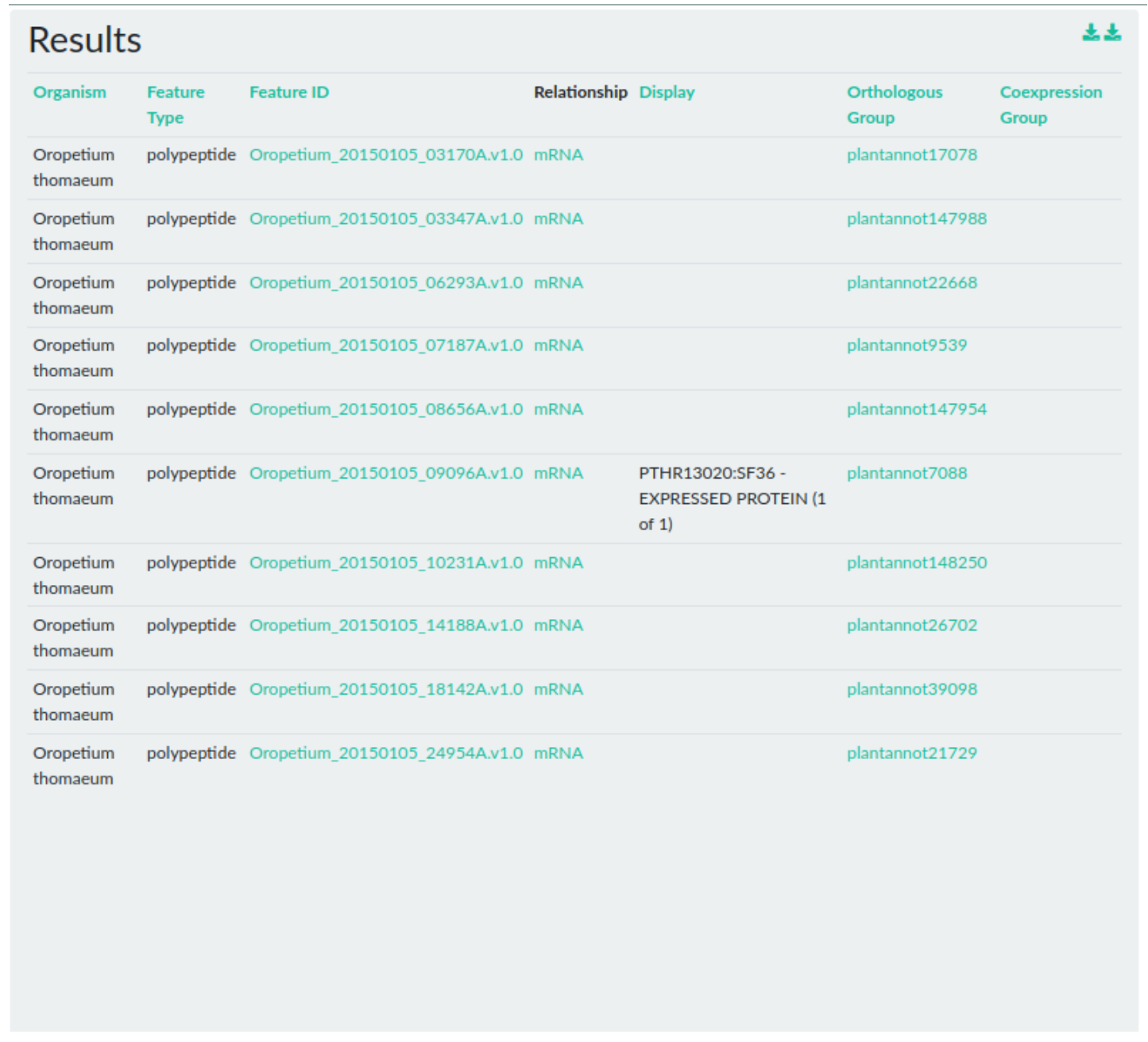

https://www.machado.cnptia.embrapa.br/plantannot/find/?

q=\&selected_facets=organism\%3AOropetium+thomaeum\&selected_facets=so_term\%3Apolype ptide\&selected_facets=orthology\%3A1\&selected_facets=orthologs_coexpression\%3Atrue\&sele cted_facets=analyses\%3Ano+diamond+matches\&selected_facets=analyses\%3Ano+interprosc an+matches 